PoS $\quad$ PROCEEDINGS

\title{
CP Violation
}

\author{
Luca Silvestrini* \\ INFN, Sez. di Roma \\ P.le A. Moro, 2 \\ 00185 Rome \\ Italy \\ E-mail: Luca.Silvestrinieromal.infn.it
}

We discuss some aspects of $\mathrm{CP}$ violation in heavy quarks, focusing on searches of New Physics

The Xth Nicola Cabibbo International Conference on Heavy Quarks and Leptons, October 11-15, 2010

Frascati (Rome) Italy

${ }^{*}$ Speaker. 


\section{Introduction}

The Standard Model (SM) of electroweak and strong interactions is extremely successful in describing all presently available experimental data. However, its validity can extend at most to energies of the order of the Planck scale, where gravity comes into play. Let us therefore consider the SM as an effective theory valid up to a scale $\Lambda$. We can then write the SM Lagrangian as

$$
\mathscr{L}=C_{2} \Lambda^{2} H^{\dagger} H+\lambda\left(H^{\dagger} H\right)^{2}+\mathscr{L}_{\text {gauge }}+\mathscr{L}_{\text {Yukawa }}+\sum_{d=5}^{\infty} \sum_{i=1}^{n_{d}} \frac{C_{d}^{i}}{\Lambda^{(d-4)}} O_{d}^{i}
$$

where $O_{d}^{i}$ is a generic gauge-invariant operator of dimension $d$. Now, it turns out that the Lagrangian truncated at $d \leq 4$ has some very important "accidental" symmetries that are violated by $O_{d>4}^{i}$. Most notable examples of such symmetries are given by baryon and lepton number conservation. The agreement of the SM with experimental data would suggest a very high value of $\Lambda$, so that the breaking of SM accidental symmetries gets strongly suppressed by the inverse powers of $\Lambda$ in front of the higher-dimensional operators. However, we see from the first term in eq. (1.1) that $C_{2} \Lambda$ controls the scale of electroweak symmetry breaking. Thus, unless we are willing to accept an extremely small value of $C_{2}$ (which means an extremely large amount of fine-tuning, since radiative corrections within the effective theory naturally generate $C_{2} \sim \mathscr{O}(1)$ ), we are forced to consider values of the New Physics (NP) scale $\Lambda$ not too far above the electroweak scale. But then the SM accidental symmetries require that NP has a peculiar structure, so that the cofficients of symmetrybreaking higher dimensional operators are strongly suppressed and the phenomenological success of the SM remains unscathed. Turning the argument around, the coefficients of those higher dimensional operators that break SM accidental symmetries provide the most stringent constraints on the NP scale and couplings (or better, on a combination thereof).

Let us now concentrate on two accidental symmetries of the SM: i) the absence of tree-level Flavour Changing Neutral Currents (FCNC), and the GIM suppression of loop-mediated FCNC; ii) The absence of tree-level $\mathrm{CP}$ violation in weak interactions. These accidental symmetries ensure that flavour physics is extremely sensitive to NP. In particular, CP violation in quark weak interactions in the SM is governed by one single phase in the Cabibbo-Kobayashi-Maskawa (CKM) mixing matrix, leading to stringent correlations between all $\mathrm{CP}$ violating observables. A very useful tool to study these correlations is given by the so-called Unitarity Triangle Analysis (UTA) [1]. The unitarity of the CKM matrix implies several triangular relations; in particular, one of these relations defines a triangle whose sides and angles are connected to several observables in $B$ physics. Since all CP violating observables are connected to the phase of the CKM matrix, they all translate into a constraint on the apex of the UT. Furthermore, the UTA allows to combine in a coherent way all constraints coming from both $\mathrm{CP}$-conserving and $\mathrm{CP}$-violating processes. For example, in Figure 1 we compare the determination of the UTA using only the measurements of the UT angles (left) or all other measurements (right). From the fit to angle measurements we obtain at $68 \%$ probability $\bar{\rho}=0.126 \pm 0.028$ and $\bar{\eta}=0.324 \pm 0.017$, while from the fit to the other observables we get $\bar{\rho}=0.131 \pm 0.028$ and $\bar{\eta}=0.387 \pm 0.021$. There is some tension between the two determinations of $\bar{\eta}$, which might be due to some small NP contribution. 

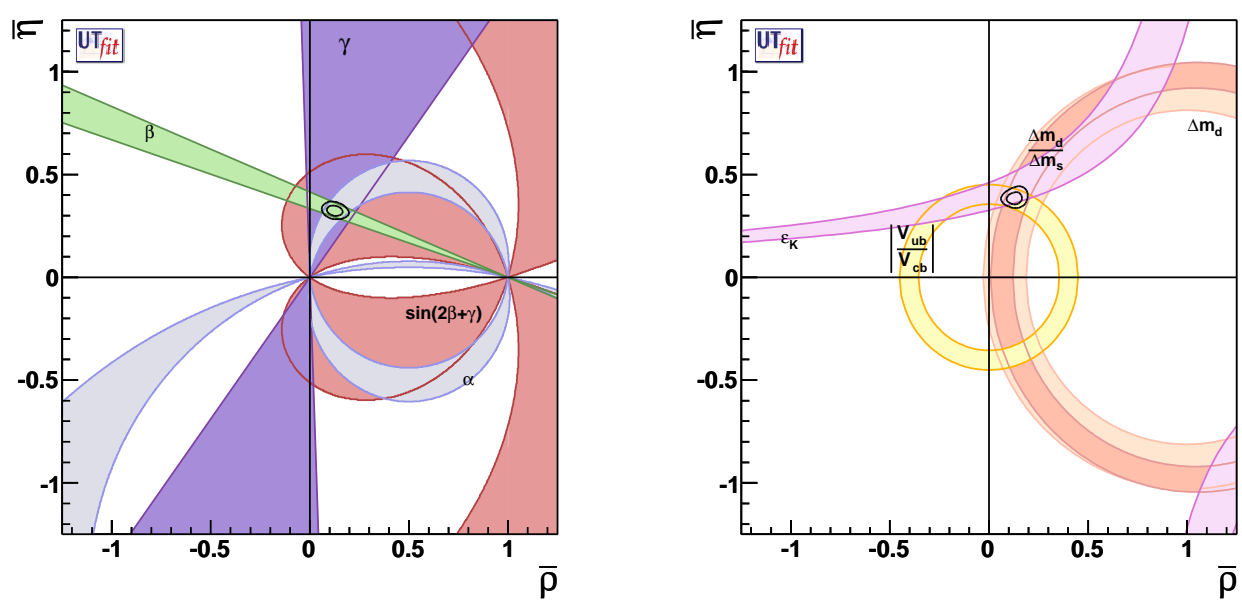

Figure 1: Determination of the UTA using only the measurements of the UT angles (left) or all other measurements (right). The contours correspond to $68 \%$ and $95 \%$ probability regions. The coloured regions correspond to $95 \%$ probability for a single constraint.

\section{CP violation in $B_{q}$ mixing}

$B_{q}$ mixing is governed by the transition matrix element between $B_{q}$ and $\bar{B}_{q}$ mesons, which can be parameterized in terms of two fundamental matrix elements: $M_{12}$, which is dominated by the exchange of virtual heavy states (top quarks and possibly new heavy particles), and $\Gamma_{12}$, which is dominated by the tree-level exchange of on-shell intermediate states. We assume here and in the following that NP is a negligible correction to tree-level processes (except for chirally suppressed decays, where a chirally-enhanced NP could compete with chirally-suppressed treelevel SM amplitudes). We can therefore write the relevant amplitudes in terms of SM ones as follows:

$$
\begin{aligned}
M_{12, q}^{\mathrm{full}} & =\left\langle B_{q}\left|\mathscr{H}_{\Delta B=2}^{\text {eff }}\right| \bar{B}_{q}\right\rangle=M_{12, q}^{\mathrm{SM}}+M_{12, q}^{\mathrm{NP}}=C_{B_{q}} e^{i \phi_{B_{q}}} M_{12, q}^{\mathrm{SM}} \\
\Gamma_{12, q}^{\text {full }} & =\Gamma_{12, q}^{\mathrm{SM}}+\text { penguin effects }
\end{aligned}
$$

with $q=d$, s. Notice that $\operatorname{Im}\left(\Gamma_{12, q}^{\mathrm{SM}} / M_{12, q}^{\mathrm{SM}}\right) \sim 0$ due to GIM suppression, since $M_{12, q}^{\mathrm{SM}} \propto\left(V_{t b} V_{t q}^{*}\right)^{2}$ and $\Gamma_{12, q}^{\mathrm{SM}} \propto\left(V_{t b} V_{t q}^{*}\right)^{2}+\mathrm{GIM}$-suppressed terms. On the other hand, in the standard CKM parameterization,

$$
\operatorname{Arg}\left(M_{12, d}^{\mathrm{SM}}\right)=2 \beta \sim \mathscr{O}(1) \quad \text { but } \quad \operatorname{Arg}\left(M_{12, s}^{\mathrm{SM}}\right)=-2 \beta_{s} \sim \mathscr{O}\left(10^{-2}\right) .
$$

From experiments we can extract the following combinations of the above parameters:

$$
\begin{aligned}
& \Delta m_{B_{q}}=2\left|M_{12, q}^{\mathrm{full}}\right|=C_{B_{q}} \Delta m_{B_{q}}^{\mathrm{SM}}, \quad \frac{\Delta \Gamma_{q}}{\Delta m_{B_{q}}}=\operatorname{Re} \frac{\Gamma_{12, q}^{\mathrm{full}}}{M_{12, q}^{\mathrm{full}}} \sim \frac{\Delta \Gamma_{q}^{\mathrm{SM}}}{\Delta m_{B_{q}}^{\mathrm{SM}}} \frac{\cos 2 \phi_{B_{q}}}{C_{B_{q}}}, \\
& A_{\mathrm{SL}}^{q}=\operatorname{Im} \frac{\Gamma_{12, q}^{\mathrm{full}}}{M_{12, q}^{\text {full }}} \sim-\frac{\Delta \Gamma_{q}^{\mathrm{SM}}}{\Delta m_{B_{q}}^{\mathrm{SM}}} \frac{\sin 2 \phi_{B_{q}}}{C_{B_{q}}} \sim-\frac{\Delta \Gamma_{q}}{\Delta m_{B_{q}}} \tan 2 \phi_{B_{q}}, \\
& S_{J / \Psi K} \sim \sin 2\left(\beta+\phi_{B_{d}}\right), \quad S_{J / \Psi \phi} \sim \sin 2\left(-\beta_{s}+\phi_{B_{s}}\right) .
\end{aligned}
$$



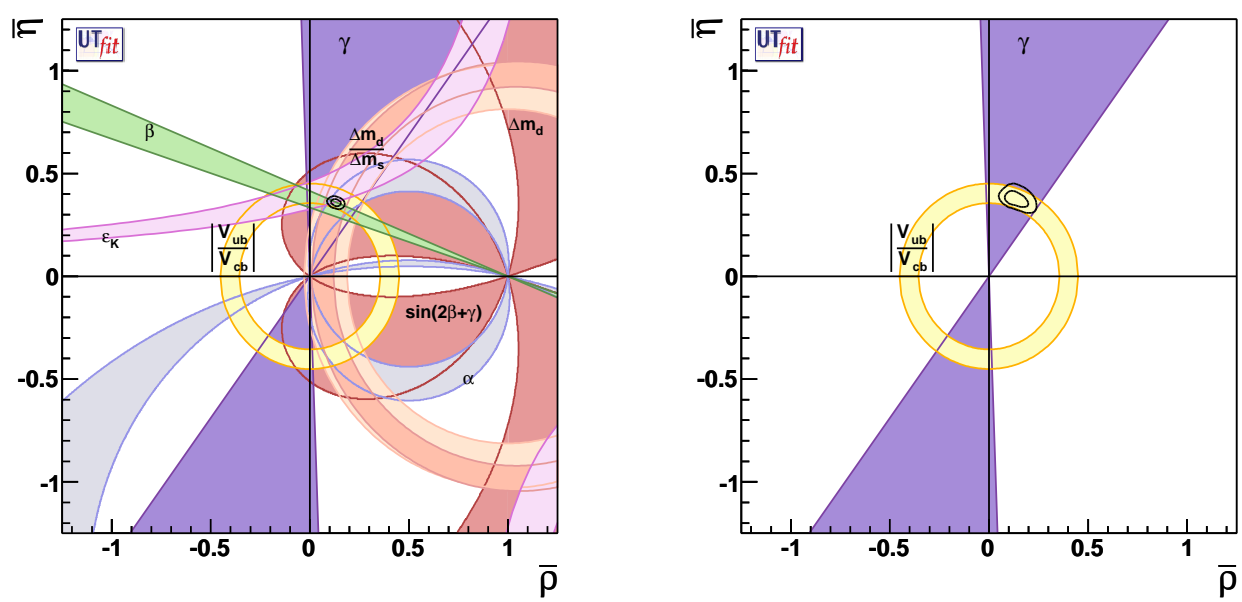

Figure 2: Determination of the UTA within the SM (left) or in the presence of NP (right). The contours correspond to $68 \%$ and $95 \%$ probability regions. The coloured regions correspond to $95 \%$ probability for a single constraint.

\begin{tabular}{|l||c|c|c|c|}
\hline Parameter & $C_{B_{d}}$ & $\left.\phi_{B_{d}}{ }^{\circ}\right]$ & $C_{B_{s}}$ & $\phi_{B_{s}}\left[^{\circ}\right]$ \\
\hline Value & $0.95 \pm 0.14$ & $-3.1 \pm 1.7$ & $0.95 \pm 0.10$ & $(-68 \pm 8) \cup(-20 \pm 8)$ \\
\hline
\end{tabular}

Table 1: Numerical results (at $68 \%$ probability) for the NP parameters in $B_{q}$ mixing.

To exploit the full constraining power of the measurements in eq. (2.3) we must obtain a NP-free determination of CKM parameters, so that we can compute the SM mixing amplitudes. To this aim, we use tree-level processes: semileptonic $B$ decays determine $\left|V_{u b}\right|$ and $\left|V_{c b}\right|, B \rightarrow D K$ decays determine the angle $\gamma$ of the Unitarity Triangle (UT) and $B \rightarrow \pi \pi, \pi \rho$ and $\rho \rho$ decays determine the angle $\alpha$ [2]. Using the tree-level UT, we can extract $C_{B_{q}}$ and $\phi_{B_{q}}$ from experimental data [2-4].

In Figure 2 we compare the determination of the UT in the presence of NP to the result of the SM UTA. Thanks to the redundancy of the UTA, the accuracy obtained in the presence of NP is comparable to the SM one.

Figure 3 shows the result of the NP analysis for the $B_{d}$ and $B_{s}$ sectors. Numerical results for NP parameters are summarized in table 1.

Given the experimental measurements, the results for $\phi_{B_{s}}$ show a discrepancy of $3.2 \sigma$ from the SM value, pointing to NP contributions with new sources of flavor violation in the transition within $2^{\text {nd }}$ and $3^{\text {rd }}$ generation. The results for $\phi_{B_{d}}$ show a slight discrepancy from the SM value by less than $2 \sigma$. As a consequence, NP contributions in transitions within $1^{\text {st }}$ and $3^{r d}$ generations are not yet excluded, but are limited to be of $\mathscr{O}(30-40 \%)$, while NP contributions in transitions within $2^{\text {nd }}$ and $3^{\text {rd }}$ generations of the order of the SM one are favoured (see Fig. 4).

Large NP contributions to $b \leftrightarrow s$ transitions arise naturally in several NP models. For example, they are expected in nonabelian flavour models, given the large breaking of flavour SU(3) by the top Yukawa coupling. In addition, supersymmetric Grand Unified Theories (SUSY-GUTs) provide a rather general connection between the large mixing angles in neutrino oscillations and large NP 

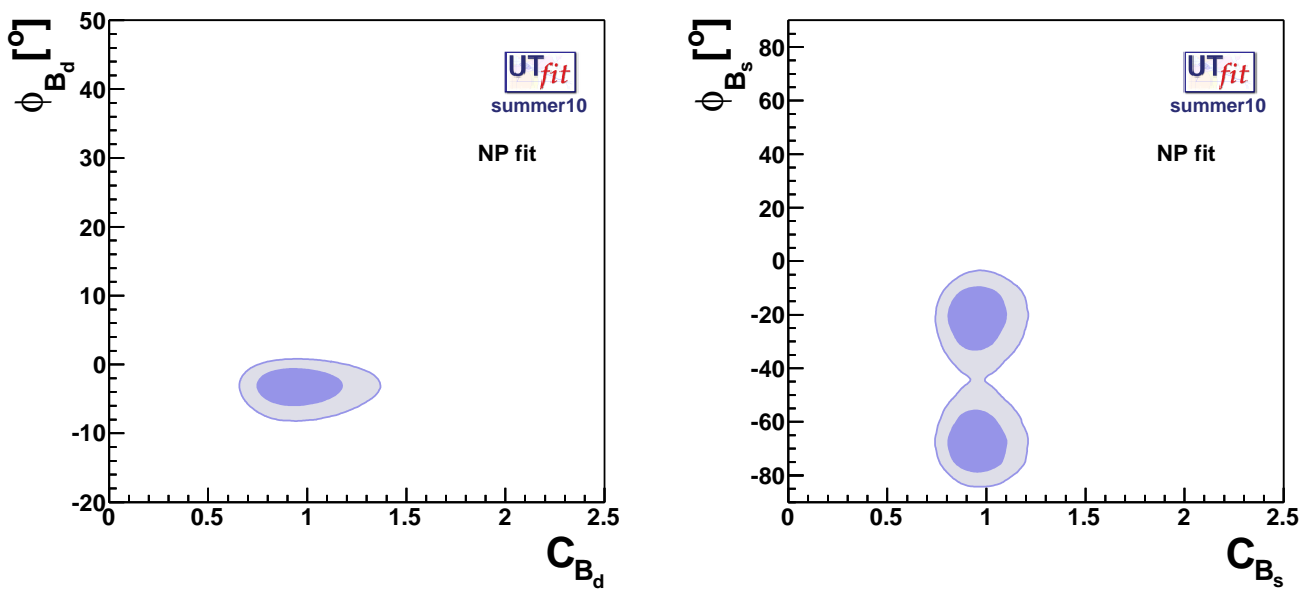

Figure 3: The dark and light colored areas show the $68 \%$ and $95 \%$ probability regions in the 2-dimensional planes $\left(C_{B_{d}}, \phi_{B_{d}}\right)$ (left) and $\left(C_{B_{s}}, \phi_{B_{s}}\right)$ (right).
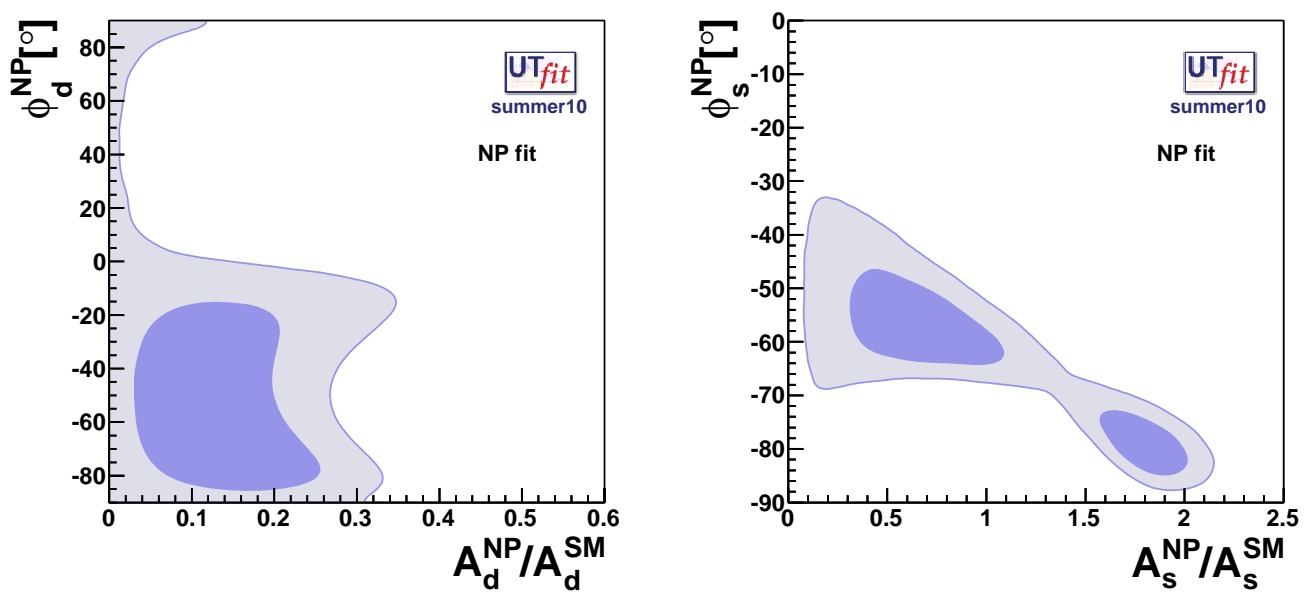

Figure 4: The dark and light colored areas show the $68 \%$ and $95 \%$ probability regions in the 2-dimensional planes $\left(A_{d}^{\mathrm{NP}} / A_{d}^{\mathrm{SM}}, \phi_{d}^{\mathrm{NP}}\right)($ left $)$ and $\left(A_{s}^{\mathrm{NP}} / A_{s}^{\mathrm{SM}}, \phi_{s}^{\mathrm{NP}}\right)$ (right).

contributions to $b \leftrightarrow s$ processes.

\subsection{NP in $B \rightarrow K \pi$}

Let us now turn to NP in $b \rightarrow s$ penguins. For reasons of space, we shall concentrate on $B \rightarrow K \pi$ decays. The difference $\Delta A_{\mathrm{CP}}=A_{\mathrm{CP}}\left(K^{+} \pi^{0}\right)-A_{\mathrm{CP}}\left(K^{+} \pi^{-}\right)$has recently received considerable attention, following the new measurement $\Delta A_{\mathrm{CP}}=0.164 \pm 0.037$ published by the Belle collaboration [5]. It has been argued that $\Delta A_{\mathrm{CP}}$ could be a hint of New Physics (NP), but alternative explanations within the Standard Model (SM) have also been considered.

To understand whether $B \rightarrow K \pi$ decays are really puzzling, possibly calling for NP, one has 

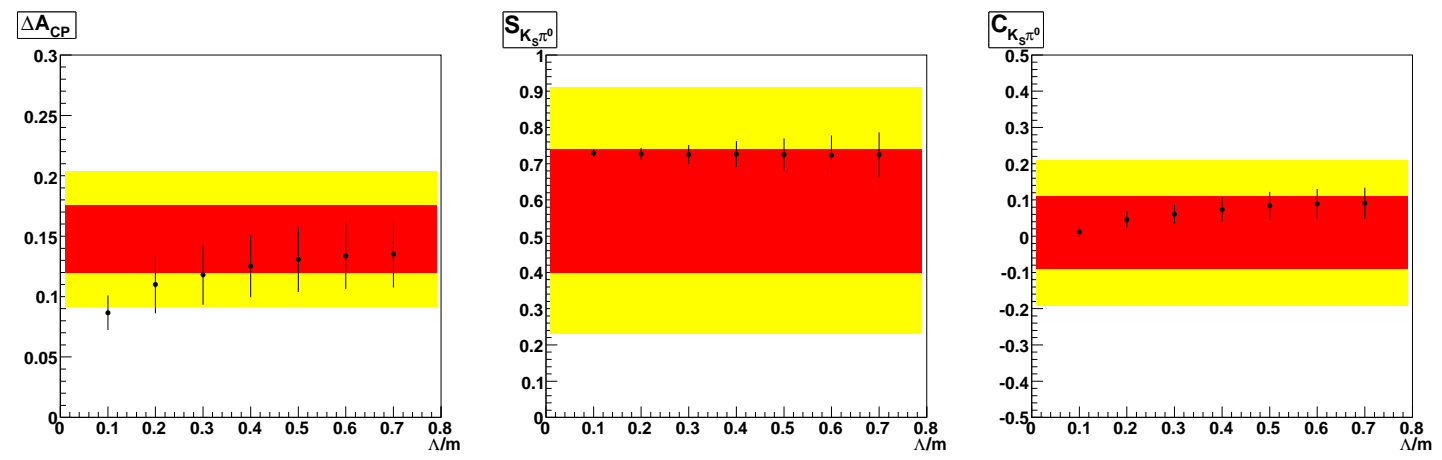

Figure 5: Some fit results as functions of the upper bound on power corrections.

to control the SM expectations for the $B \rightarrow K \pi$ amplitudes with a level of accuracy dictated by the size of the potential NP contributions. Thanks to the progress of theory in the last few years, we know that two-body non-leptonic $B$ decay amplitudes are factorizable in the infinite $b$-quark mass limit, i.e. computable in terms of a reduced set of universal non-perturbative parameters [6-8]. However, the accuracy of the predictions obtained with factorization is limited by the uncertainties on the non-perturbative parameters on the one hand and by the uncalculable subleading terms in the $1 / m_{b}$ expansion on the other. The latter problem is particulary severe for $B \rightarrow K \pi$ decays where some power-suppressed terms are doubly Cabibbo-enhanced with respect to factorizable terms [9]. Indeed factorization typically predicts too small $B \rightarrow K \pi$ branching ratios, albeit with large uncertainties. The introduction of subleading terms, certainly present at the physical value of the $b$ quark mass, produces large effects in branching ratios and $\mathrm{CP}$ asymmetries, leading to a substantial model dependence of the SM predictions. Given this situation, NP contributions to $B \rightarrow K \pi$ amplitudes could be easily misidentified.

In Fig. 5 we display the dependence of the SM fit results on the size of power corrections for some $B \rightarrow K \pi \mathrm{CP}$ asymmetries [10]. We see that $\Delta A_{\mathrm{CP}}$ can be reproduced within the SM for power corrections to factorization of the order of $30 \%$, while the coefficients of the time-dependent $\mathrm{CP}$ asymmetry in $B \rightarrow K_{s} \pi^{0}$ are almost insensitive to power corrections and might therefore provide a test of the SM with improved experimental results. Unfortunately, at present the situation is inconclusive: the observed value of $\Delta A_{\mathrm{CP}}$ could be given by NP (in particular, by new sources of $\mathrm{CP}$ violation in $b \rightarrow s$ electroweak penguins), but it can also be explained within the SM due to uncalculable power corrections to factorization.

\section{Conclusions}

$\mathrm{CP}$ violating processes are a very powerful probe of NP. Using all presently available experimental data, we can constrain NP contributions to CP violation in $s \rightarrow d$ and $b \rightarrow d$ transitions to be at the level of $30-40 \%$, while the possibility of $\mathscr{O}(1) \mathrm{NP}$ contributions to $b \rightarrow s$ transitions is still open. Forthcoming experimental data from the TeVatron and LHCb will soon clarify if (and at what level) NP is manifesting itself in $B_{s}$ mixing.

L.S. is associated to the Dipartimento di Fisica, Università di Roma "La Sapienza". 


\section{References}

[1] C. Tarantino, these proceedings.

[2] M. Bona et al. [UTfit Collaboration], JHEP 0603 (2006) 080 [arXiv:hep-ph/0509219].

[3] M. Bona et al. [UTfit Collaboration], Phys. Rev. Lett. 97 (2006) 151803 [arXiv:hep-ph/0605213]; JHEP 0803 (2008) 049 [arXiv:0707.0636 [hep-ph]].

[4] M. Bona et al. [UTfit Collaboration], PMC Phys. A3 (2009) 6 [arXiv:0803.0659 [hep-ph]].

[5] [The Belle Collaboration], Nature 452 (2008) 332.

[6] M. Beneke, G. Buchalla, M. Neubert and C. T. Sachrajda, Phys. Rev. Lett. 83 (1999) 1914 [arXiv:hep-ph/9905312]; Nucl. Phys. B 591 (2000) 313 [arXiv:hep-ph/0006124]; M. Beneke and M. Neubert, Nucl. Phys. B 651 (2003) 225 [arXiv:hep-ph/0210085]; M. Beneke and S. Jager, Nucl. Phys. B 751 (2006) 160 [arXiv:hep-ph/0512351]; Nucl. Phys. B 768 (2007) 51 [arXiv:hep-ph/0610322].

[7] H. n. Li and H. L. Yu, Phys. Rev. Lett. 74 (1995) 4388 [arXiv:hep-ph/9409313]; Phys. Lett. B 353 (1995) 301; Phys. Rev. D 53 (1996) 2480 [arXiv:hep-ph/9411308].

[8] C. W. Bauer, D. Pirjol and I. W. Stewart, Phys. Rev. D 65 (2002) 054022 [arXiv:hep-ph/0109045]; C. W. Bauer, S. Fleming, D. Pirjol, I. Z. Rothstein and I. W. Stewart, Phys. Rev. D 66 (2002) 014017 [arXiv:hep-ph/0202088]; C. W. Bauer, D. Pirjol and I. W. Stewart, Phys. Rev. Lett. 87 (2001) 201806 [arXiv:hep-ph/0107002]; J. g. Chay and C. Kim, Phys. Rev. D 68 (2003) 071502 [arXiv:hep-ph/0301055]; Nucl. Phys. B 680 (2004) 302 [arXiv:hep-ph/0301262].

[9] M. Ciuchini, E. Franco, G. Martinelli and L. Silvestrini, Nucl. Phys. B 501 (1997) 271 [arXiv:hep-ph/9703353]; M. Ciuchini, R. Contino, E. Franco, G. Martinelli and L. Silvestrini, Nucl. Phys. B 512 (1998) 3 [Erratum-ibid. B 531 (1998) 656] [arXiv:hep-ph/9708222]; Nucl. Instrum. Meth. A 408 (1998) 28 [arXiv:hep-ph/9801420]; C. Isola, M. Ladisa, G. Nardulli, T. N. Pham and P. Santorelli, Phys. Rev. D 64 (2001) 014029 [arXiv:hep-ph/0101118]; M. Ciuchini, E. Franco, G. Martinelli, M. Pierini and L. Silvestrini, Phys. Lett. B 515 (2001) 33 [arXiv:hep-ph/0104126]; C. Isola, M. Ladisa, G. Nardulli, T. N. Pham and P. Santorelli, Phys. Rev. D 65 (2002) 094005 [arXiv:hep-ph/0110411]; M. Ciuchini, E. Franco, G. Martinelli, M. Pierini and L. Silvestrini, arXiv:hep-ph/0208048; C. Isola, M. Ladisa, G. Nardulli and P. Santorelli, Phys. Rev. D 68 (2003) 114001 [arXiv:hep-ph/0307367]; P. Zenczykowski, Phys. Lett. B 590 (2004) 63 [arXiv:hep-ph/0402290]; M. Ciuchini, E. Franco, G. Martinelli, A. Masiero, M. Pierini and L. Silvestrini, arXiv:hep-ph/0407073.

[10] M. Ciuchini, E. Franco, G. Martinelli, M. Pierini, L. Silvestrini, Phys. Lett. B674 (2009) 197-203. [arXiv:0811.0341 [hep-ph]]. 